\title{
NuSTAR: System engineering and modeling challenges in pointing reconstruction for a deployable $\mathrm{X}$-ray telescope
}

\author{
D. Isaiah Harp ${ }^{* a}$, Carl Christian Liebe ${ }^{\mathrm{a}}$, William Craig ${ }^{\mathrm{b}}$, Fiona Harrison ${ }^{\mathrm{c}}$, Kristin Kruse-Madsen ${ }^{\mathrm{c}}$, \\ Andreas Zoglauer \\ a Jet Propulsion Laboratory, California Institute of Technology \\ 4800 Oak Grove Dr.; Pasadena, CA 91109-8099, USA \\ ${ }^{\mathrm{b}}$ University of California, Berkeley, Space Sciences Laboratory \\ 7 Gauss Way; Berkeley, Ca 94720-7450, USA \\ ${ }^{c}$ Space Radiation Laboratory, California Institute of Technology \\ 290-17 Cahill; 1200 E. California Blvd.; Pasadena, CA 91125, USA
}

\begin{abstract}
The Nuclear Spectroscopic Telescope Array (NuSTAR) is a NASA Small Explorer mission that will make the first sensitive images of the sky in the high energy X-ray band $(6-80 \mathrm{keV})$. The NuSTAR observatory consists of two coaligned grazing incidence hard X-ray telescopes with a $\sim 10$ meter focal length, achieved by the on-orbit extension of a deployable mast.

A principal science objective of the mission is to locate previously unknown high-energy X-ray sources to an accuracy of 10 arcseconds (3-sigma), sufficient to uniquely identify counterparts at other wavelengths. In order to achieve this, a star tracker and laser metrology system are an integral part of the instrument; in conjunction, they will determine the orientation of the optics bench in celestial coordinates and also measure the flexures in the deployable mast as it responds to the varying on-orbit thermal environment, as well as aerodynamic and control torques. The architecture of the NuSTAR system for solving the attitude and aspect problems differs from that of previous X-ray telescopes, which did not require ex post facto reconstruction of the instantaneous observatory alignment on-orbit.

In this paper we describe the NuSTAR instrument metrology system architecture and implementation, focusing on the systems engineering challenges associated with validating the instantaneous transformations between focal plane and celestial coordinates to within the required accuracy. We present a mathematical solution to photon source reconstruction, along with a detailed error budget that relates component errors to science performance. We also describe the architecture of the instrument simulation software being used to validate the end-to-end performance model.
\end{abstract}

\section{THE NUSTAR INSTRUMENT}

The integrated NuSTAR observatory consists of a single instrument rigidly mounted to a spacecraft bus, based on the LEOStar- $2^{\mathrm{TM}}$ design ${ }^{1}$, containing the avionics, power, and attitude control systems. The instrument itself is made up of two benches, one for the optics and one for the focal plane, which are coupled during launch, separation, and initial checkout by non-explosive actuators and separated by a deployable mast once on orbit.

The optical bench is a highly rigid carbon composite truss structure with near-zero CTE. It holds two conicalapproximation Wolter-I optics which are manufactured by concentrically assembling slumped glass shells onto an inner titanium mandrel, with epoxied graphite spacers in between. Before placement, the glass sectors are coated with mirrored multilayers of Platinum and Carbon $(\mathrm{Pt} / \mathrm{C})$ or Tungsten and Silicon (W/Si), for optimizing X-ray reflectance at the radius and graze angle of the shell. ${ }^{2}$ To first order the optics behave much like thin lenses; hence, small tips and tilts of an optic or its bench reduce throughput but do not translate the focal point in the focusing plane.

\footnotetext{
*daniel.i.harp@jpl.nasa.gov; phone 818393 6708; fax 8183930068
}

Modeling, Systems Engineering, and Project Management for Astronomy IV, edited by George Z. Angeli, Philippe Dierickx, Proc. of SPIE Vol. 7738, 77380Z

(c) 2010 SPIE · CCC code: $0277-786 \mathrm{X} / 10 / \$ 18 \cdot$ doi: $10.1117 / 12.856626$ 
The focal plane bench is an aluminum honeycomb sandwich structure. The focal plane modules housing the detectors and their electronics mount directly to the bench. The detectors themselves are cadmium zinc telluride (CdZnTe) semiconductors, and are shielded by an active veto cesium iodide (CsI) crystal. Also mounted on the focal plane bench is a canister containing the deployable mast in its stowed configuration.

When deployed, the mast achieves a focal length of $10150 \mathrm{~mm}$. While highly rigid in this configuration, thermal gradients in the mast nonetheless induce non-negligible relative motion between the two benches. Analysis predicts that, in the worst-case thermal conditions, the size of this motion would cause a vector projected from one bench to another to be displaced by about $1.5 \mathrm{~mm}$. If there were no compensation for this effect, such a motion would blur a point source by nearly 30 arcseconds. As this measure exceeds the level 1 requirement on source localization, the instrument requires a metrology system to compensate for the motion. This system is conceptually described in the next section. A rendering of the NuSTAR observatory is shown in Figure 1.

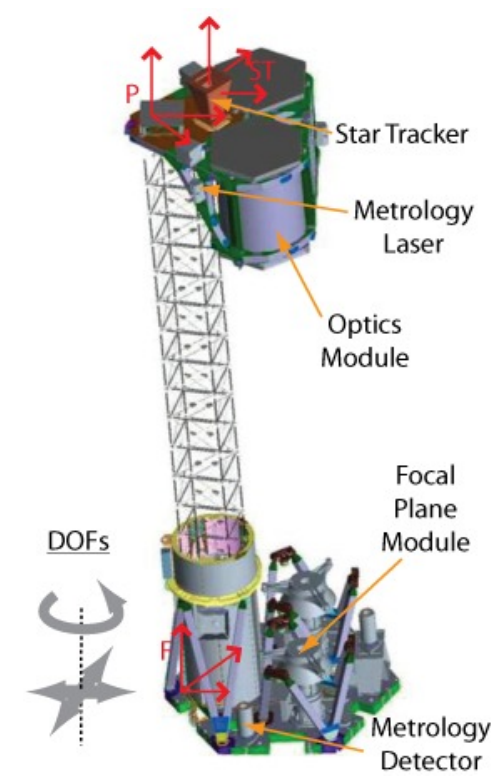

Figure 1. Rendering of the NuSTAR observatory.

\section{THE METROLOGY SYSTEM}

\subsection{Description}

The metrology system includes both the star tracker and the two laser-detector pairs illustrated in Figure 1. The star tracker is rigidly mounted to the optical bench and, as such, solves for the complete 3-degree of freedom (DOF) rotation from the celestial coordinate system to that bench; that is, the star tracker provides the attitude of the optical bench.

There is also a 6-DOF aspect transformation between the two benches. The metrology lasers are not required to measure all 6 DOFs. The longitudinal distance (along the axis of the mast) between the two benches is $\sim 10$ meters and thermal variations predicted in this direction would introduce an error of less than $\sim 0.1$ arcseconds in the error budget. This is negligible, and so deviations in the distance between the benches are not tracked. Also, the metrology lasers do not measure the angle of the focal plane bench relative to the optical bench (i.e. deviations from the nominal parallelism of the benches). Note that this does not imply that the absolute tip and tilt of the optics bench with respect to the stars is ignored. All such motion is detected by the optics bench mounted star tracker. The metrology lasers then measure translation in 2 axes (those directions transverse to the laser beams) and a clocking angle (rotation about the mast axis). Within the aspect transformation between the two benches, only these 3 DOFs have the potential to introduce errors large enough that they must be measured. The motions at the focal plane bench due to these DOFs are shown with arrows in Figure 1. 
A metrology system with the described features can be implemented with a star tracker on either bench and any two independent fiducials tracked between the benches. NuSTAR has chosen to locate the star tracker on the outboard bench, so it can be co-boresighted with the telescopes, which is the optimal configuration. ${ }^{3}$ The fiducial tracking could either be implemented with two laser pointers on the optical bench illuminating 2 focal planes on the focal plane bench, or with two light sources (e.g. LEDs) on the focal plane bench being imaged by two target tracking cameras mounted on the optics bench. NuSTAR selected the former architecture. In this configuration the focal plane does not have optics in front of it, simplifying implementation. The disadvantage of this architecture is the high demands on the beam pointing stability of the metrology lasers.

\subsection{Implementation}

The detectors for the metrology lasers are based on a $20 \mathrm{~mm}$ Position Sensing Detector (PSD). The two-dimensional PSD is a large photodiode that is able to detect the centroid of a light spot falling on its surface in two dimensions. It is possible to get PSD chips with dimensions up to $45 \mathrm{~mm}$, but a trade study on the maximum travel distance for the mast determined that only a $\sim 20 \mathrm{~mm}$ PSD chip was required. The operation range requirement is that the metrology system shall be able to operate over the range of $\sim 6 \mathrm{~mm}$ in any one axis $(+/-\sim 3 \mathrm{~mm})$.

To attenuate the background, a narrow bandpass filter is mounted in front of the PSD detector. Also, the metrology detector has a stray light baffle and neutral density filter. However, there are still residual background signals that may disturb the measurement. To remove these signals from, e.g., stray light on the baffle knives, dark current, the moon in the field of view, etc., the laser beam is turned off 4 times a second and a background measurement is made. The latest background signal measurement is always subtracted from a laser signal measurement.

The metrology laser is focused to a spot of a few millimeters on a PSD detector that is located at a distance of $\sim 10$ meters from the metrology laser. This is achieved utilizing a single aspherical lens. ${ }^{4}$

\section{PHOTON SOURCE RECONSTRUCTION}

The integration of a metrology system in the instrument was an architectural decision to enable the correction of the arrival position of a detected x-ray photon, in order to account for the bench-to-bench (aspect) motion of the instrument. The following presents one possible resolution to the mathematical form of that correction, even in the presence of errors due to imperfect deployment and cool-down on orbit.

\subsection{Statement of the Problem}

Consider a celestial coordinate system in J2000, and a coordinate system on the surface of a detector array. The problem of determining from where in the sky a registered photon originated is equivalent to solving for the transformation between detector coordinates and celestial coordinates at the instant of photon arrival.

It will be shown that for an arbitrary instant this transformation can be found using the lasers and star tracker, provided that the locations and alignments of metrology components relative to the benches to which they mount are known. However, because of biases introduced by launch and on-orbit cool-down, these alignments are not precisely known when the instrument is first powered on.

To find that initial configuration, the first observation performed by the observatory will be of a stable X-ray point source: either the Crab nebula or the quasar 3C 273. During this observation the celestial coordinates of the source are known, so it is possible to calculate a transformation between the focal plane and the sky directly. Then the alignments of the metrology components can be backed out by comparing the known transformation to the one predicted by metrology and optimizing a cost function.

Thus, there are in fact two separate problems. The X-ray alignment observation (XAO) is solved once for initial conditions (in terms of alignment). Then, it is assumed that all component alignments are static and the reconstruction problem is solved for all future instants. Here the two problems are presented in reverse order, as the XAO problem employs the formalism of the simpler reconstruction problem. 


\subsection{Formalism}

The inputs to the reconstruction problem for the NuSTAR observatory are illustrated in Figure 2. There are shown for simplicity only the 4 main coordinate systems of interest. The I- (inertial) system replaces celestial coordinates at any instant and should be thought of as co-originated with the detector coordinate system; the ST-system is the star tracker's internal coordinate system; the $\mathbf{P}$-system is originated at the equivalent optics pinhole (the virtual center of the optic in a thin lens approximation) and its axes align rigidly with the bench; the $\mathbf{F}$-system is originated at and aligns rigidly with a focal plane detector. ${ }^{*}$ Those coordinate systems indexed to the instrument are also sketched in Figure 1.

The parameters for the problem can be divided into time-dependent quantities measured on orbit, and the static alignment quantities optimized during the XAO.

Comprising the former are: the star tracker quaternion operator $\mathrm{R}_{\mathrm{ST}-\mathrm{I}}$, which is the 3-DOF rotation from the star tracker coordinate system to inertial space (celestial coordinates); the in-plane locations in F-coordinates of the laser impingements on the metrology detectors ${ }^{\dagger}, \mathbf{d}_{\mathbf{0}, \mathbf{F}}$ and $\mathbf{d}_{\mathbf{1 , F}}$; and the measured $\mathrm{x}$-ray impingements $\mathbf{e}_{\mathbf{F}}$. These quantities are recorded at different rates on different clocks; however, it is assumed here that sufficiently high sampling rates prevent the introduction of significant error from an interpolation of the data to the instant of photon arrival. Comprising the alignment quantities are: $\mathbf{c}_{\mathbf{0}, \mathbf{P}}$ and $\mathbf{c}_{\mathbf{1}, \mathbf{P}}$, the vectors to the metrology lasers; $\mathbf{m}_{\mathbf{0}, \mathbf{P}}$ and $\mathbf{m}_{\mathbf{1}, \mathbf{P}}$, pointing vectors along the boresights of those lasers; ${ }^{*} \mathrm{R}_{\mathrm{P}-\mathrm{ST}}$, the rotation from the optics bench system to the star tracker; $\mathbf{A}$, the direction of an optical axis, and $M$, the assumed mast length.

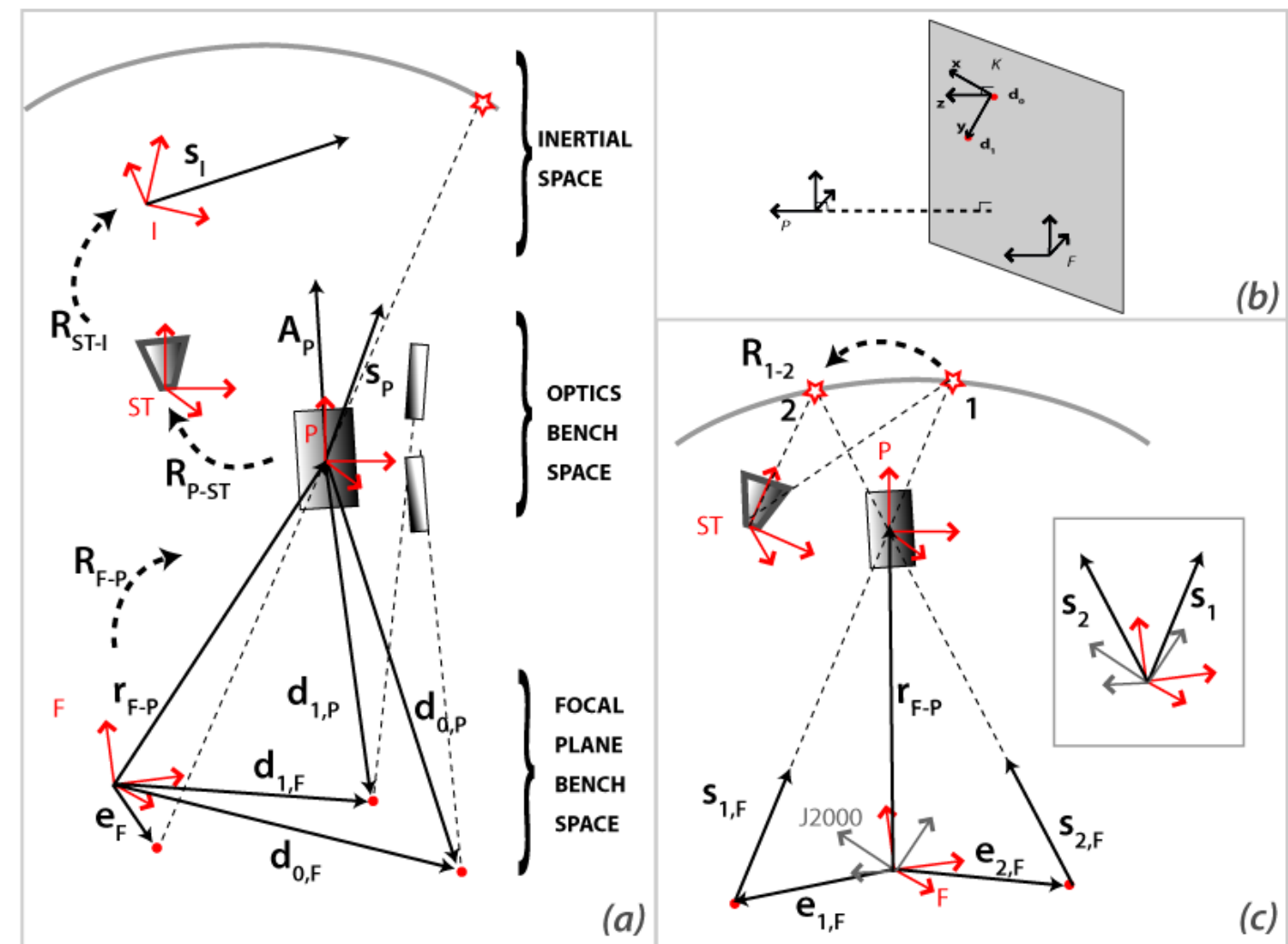

Figure 2. Sketches of (a) The observatory coordinate systems and vectors for the reconstruction problem, (b) the construction of the K-system, and (c) the coordinate systems and vectors for the XAO problem

\footnotetext{
* Note that only one optic and detector pair is analyzed. The two telescopes simply co-add on the ground, and the solution for the second is mathematically equivalent to the solution for the first.

${ }^{\dagger}$ Here, it is assumed that laser impingement and x-ray photon collection are both registered in an $\mathbf{F}$ system. This is not necessary. It is only necessary that all detector faces are known (in 6-DOF) relative to the $\mathbf{F}$ system. For simplicity, we have omitted the calibration vectors that express that knowledge, but their incorporation is mathematically trivial.

${ }^{\ddagger}$ The vectors $\mathbf{c}$ and $\mathbf{m}$ are not shown in Figure 3, but their relationship to the illustrated vectors $\mathbf{d}$ is elaborated in 3.4.
} 


\subsection{Kinematic Assumptions}

Since $\mathrm{R}_{\mathrm{P}-\mathrm{ST}}$ is static at any moment, the attitude of the optical bench is solved inertially by the star tracker, so the remaining six dynamic degrees of freedom in the solution consist of those motions of the focal plane bench relative to the optical bench. Engineering justifications were presented in section 2.1 for discarding three of those degrees of freedom based on error size analysis.

The monitored motions are the $x-y$ translations of the focal plane bench, and rolls about the mast axis. Neither of these motions alters the parallelism of the $\mathrm{x}-\mathrm{y}$ plane of $\mathbf{F}$ relative to $\mathbf{P}$. Hence, the fundamental kinematic assumption is that $\hat{i}_{F} \times \hat{j}_{F}=\hat{i}_{P} \times \hat{j}_{P}$.

\subsection{Resolution of the Reconstruction Problem ${ }^{\S}$}

As a result of the foregoing, at all moments in time the normal to the $\hat{i}_{F}-\hat{j}_{F}$ plane is well-defined in both the $\mathbf{F}$ and $\mathbf{P}$ systems. Call this normal the z-axis of a new coordinate system $\mathbf{K}$, composed of unit vectors $(\hat{x}, \hat{y}, \hat{z})$. Then, $\hat{z}_{F}=\left(\begin{array}{lll}0 & 0 & 1\end{array}\right)_{F}=\hat{z}_{P}=\left(\begin{array}{lll}0 & 0 & 1\end{array}\right)_{P}$.

Define the origin of $\mathbf{K}$ to lie at the centroid of the laser spot $\mathbf{d}_{\mathbf{0}, \mathbf{F}}$. With respect to the focal plane bench this spot is moving, due primarily to thermal motion of the mast, but it is static with respect to the optics bench. Define the y-axis of the $\mathbf{K}$ system to lie along the vector between the two laser spots. In the $\mathbf{F}$ system, this axis can be specified immediately; to wit, $\vec{y}_{F}=\vec{d}_{1, F}-\vec{d}_{0, F}$. In that expression, the $\vec{d}_{i, F}$ are measured by the metrology detector. However, using the static values of $\mathbf{c}, \mathbf{m}$, and the assumption that the mast length is $M$, the locations of the laser impingements can also be found in the $\mathbf{P}$ system. In particular, $\vec{d}_{i, P}=\vec{c}_{i}+t \cdot \vec{m}_{i}$, where $t \cdot m_{i}^{z}=\left(M+c_{i}^{z}\right)$. Then, $\vec{y}_{P}=\vec{d}_{1, P}-\vec{d}_{0, P}$. The $\mathrm{x}$-axis of the $\mathbf{K}$ system completes the right-hand set.

Since the coordinate axes of the $\mathbf{K}$ system can be specified in both the $\mathbf{F}$ and $\mathbf{P}$ systems, a direction cosine matrix rotation between the $\mathbf{F}$ and the $\mathbf{K}$, and the $\mathbf{P}$ and the $\mathbf{K}$ systems can be written in terms of the unit vectors along those axes. The rotation between the two benches can then be found immediately by matrix multiplication, $R_{F-P}=R_{K-P} \cdot R_{K-F}^{-1}$.

This matrix, $R_{F-P}$, entails all the rotation between the two coordinate systems, but what about relative displacement of the origins? Consider the 6-DOF transformation between the benches. For an arbitrary vector in F-coordinates, it takes the form:

$$
\vec{v}_{P}=T_{F-P}\left(\vec{v}_{F}\right)=\vec{r}_{P-F}+R_{F-P} \vec{v}_{F},
$$

where $\vec{r}_{P-F}$ is the vector displacement between the origins. Now, in place of an arbitrary vector, substitute the centroid of the measured metrology spots in F-coordinates, denominated $\left\langle\vec{d}_{F}\right\rangle$. Since the centroid is also known in Pcoordinates, the solution for the missing translation can be found:

$$
\vec{r}_{P-F}=<\vec{d}_{P}>-R_{F-P}<\vec{d}_{F}>
$$

At this point it is possible to find the pointing direction towards the source, $\mathbf{s}$, of the registered event, by using the vector relationships in Figure 2. Noting that the vector $\vec{r}_{P-F}$ by definition terminates at the effective optics pinhole,

$$
\vec{s}_{F}=\vec{r}_{F-p}-\vec{e}_{F}
$$

The inertial pointing to the source is then immediate:

$$
\hat{s}_{I}=R_{S T-I} \cdot R_{P-S T} \cdot R_{F-P} \cdot \hat{s}_{F} .
$$

\footnotetext{
$\S$ The first part of this section is a modification of Horn's resolution of the photogrammetric absolute orientation problem in [5].
} 
Since right ascension and declination in J2000 can be determined trivially from an inertial pointing vector, the localization of the celestial coordinate source of an event registered at the focal plane has been determined. However, it is also vital for throughput calibration to know at what angular distance off the optical axis the source lies. The cosine of that angular distance equals the inner product of $\mathbf{A}$ and $\mathbf{s}$.

$$
\xi=\cos ^{-1}\left(\vec{A}_{P} \cdot \vec{s}_{P}\right) .
$$

\subsection{The XAO Problem}

When the observatory is launched, it is expected that a number of very small displacements of components will occur. For example, the metrology detector will slightly shift relative to the metrology detector mounting feet, and the orientation of the star tracker may shift relative to the metrology lasers. Evidently, such shifts will alter the values of the alignment quantities $\mathbf{d}_{\mathbf{0}, \mathbf{P}}, \mathbf{d}_{\mathbf{1}, \mathbf{P}}, \mathrm{R}_{\mathrm{P}-\mathrm{ST}}$, and $\mathbf{A}$ that were used in the algorithms of section 3.4 from their values measured on-ground.

The XAO is performed to initialize these alignment values. Formally, the XAO treats the photon path from a source in the sky in a manner analogous to a metrology laser path between the benches. Figure 2c illustrates this statement. X-ray photons from each of two sources pass through the effective optics pinhole and are detected on the focal plane. Two vectors can then be drawn: one from each X-ray detection towards the optic. Since the source of the photons in inertial coordinates is presumed known, these two vectors are sufficient to calculate the rotation between the $\mathbf{F}$ - and $\mathbf{I}$-frames.

However, to find the vectors $\mathbf{s}_{\mathbf{i}, \mathbf{F}}$ the vector to the optics pinhole, $\mathbf{r}_{\mathbf{F}-\mathbf{P}}$, is required. That vector in turn is a function of the unknown alignments of the metrology system (in addition to the measured metrology data* ${ }^{* *}$. Call that set of alignment vectors $\{\vec{u}\}$. Begin by guessing that the alignment vectors are equivalent to the values that were measured on-ground, $\left\{\vec{u}^{(0)}\right\}$. Then for an XAO observation, $\vec{s}_{i, F}^{(0)}=f\left(\vec{e}_{i, F},\left\{\vec{u}^{(0)}\right\}\right)$ using the algorithms of section 3.4.

With those vectors, the transformation between $\mathbf{F}$ and $\mathbf{I}$ can be predicted, $T_{F-I}^{(0)}$. Observe that

$$
T_{F-I}^{(0)}=T_{S T-I} T_{P-S T}^{(0)} T_{F-P}^{(0)} ; \quad \text { where } T_{F-P}^{(0)}=f\left(\left\{u^{(0)}\right\}\right)
$$

which can be used to initially predict the unknown transformation $T_{P-S T}^{(0)}$, which is the alignment of the star tracker to its bench. This transformation and the vectors $\left\{\vec{u}^{(0)}\right\}$ fully characterize the observatory configuration.

Next, this configuration is assumed and the known $\vec{e}_{i, F}$ are ignored. Instead, their values are predicted using the known point source location and the assumed configuration; here, $\vec{e}_{i, F}^{(0)}=f\left(\vec{s}_{i, I}, T_{S T-I}, T_{P-S T}^{(0)},\left\{\vec{u}^{(0)}\right\}\right)$, using the algorithms developed in 3.4. This prediction of course will differ from the actual locations of X-ray photon arrival. That is, generally there is some error on each prediction $\varepsilon_{i}=\left\|\vec{e}_{i, F}-\vec{e}_{i, F}^{(0)}\right\|>0$.

Given this error, the guesses on the alignment vectors are incremented to $\left\{\vec{u}^{(1)}\right\}$ and the error reevaluated. For a number of such iterations $n$, a cost function can be defined for the number of photons counted along $i$ :

$$
\operatorname{cost}\left(\left\{u^{(n)}\right\}\right)=\sum_{\mathrm{i}} \varepsilon_{i}^{(n)}
$$

This function can be minimized with a number of standard algorithms in order to yield best values of both the alignment vectors $\{\vec{u}\}$ and the alignment of the star tracker $T_{P-S T}$. In principle, it is possible to fully constrain the configuration with just two photons, as only two vectors are needed to determine the transformation between $\mathbf{F}$ and $\mathbf{I}$. However, in actuality thousands of X-ray photons will be used.

\footnotetext{
** In what follows, to simplify the notation it is assumed that the data registered on the metrology detector, $\vec{d}_{i, F}$, is always read out when needed, and is not shown explicitly.
} 
Also note that it is not necessary that the photons come from different places in the sky (as drawn). Instead, the observatory can simply slew to two different pointing directions with the source in the FOV. The change in the star tracker quaternion between the two observations can be used to solve for the effective $\mathrm{R}_{1-2}$, the inertial transformation that would exist between vectors to the sources if they were viewed in a simultaneous observation.

In practice, the observatory will do a full raster scan of the known source. With this data, it is also possible to experimentally determine the focal length of the observatory, which is a basic assumption in these algorithms.

\section{POINTING BUDGET}

\subsection{Overview}

The foregoing has specified an algorithm $\boldsymbol{f}$ by which a pixel $(n, m)$ at any instant in time may be transformed into right ascension and declination, using metrology data measured on-orbit and another set optimized during the $\mathrm{X}$-ray alignment observation at $\mathrm{t}_{0}$.

$$
(\alpha, \delta)_{t}=f\left(\{\text { pixel }\}_{t},\{\text { metrology }\}_{t},\{X A O\}_{t=t_{0}}\right)
$$

Consider that none of the measured and optimized data are ever known without error. Each datum is subject to the thermo-structural, electronic and data collection errors of its corresponding component during measurement; alignment data are additionally subject to errors associated with the optimization algorithms themselves. There are performance requirements levied on the observatory subsystems and on the alignment and calibration process that limit the size of these errors.

However, all these errors create inaccuracies in photon reconstruction. The NuSTAR pointing budget was an early ${ }^{\dagger \dagger}$ spreadsheet model of how those errors accumulate. It was also used to generate requirements for the subsystems in the early definition of the observatory.

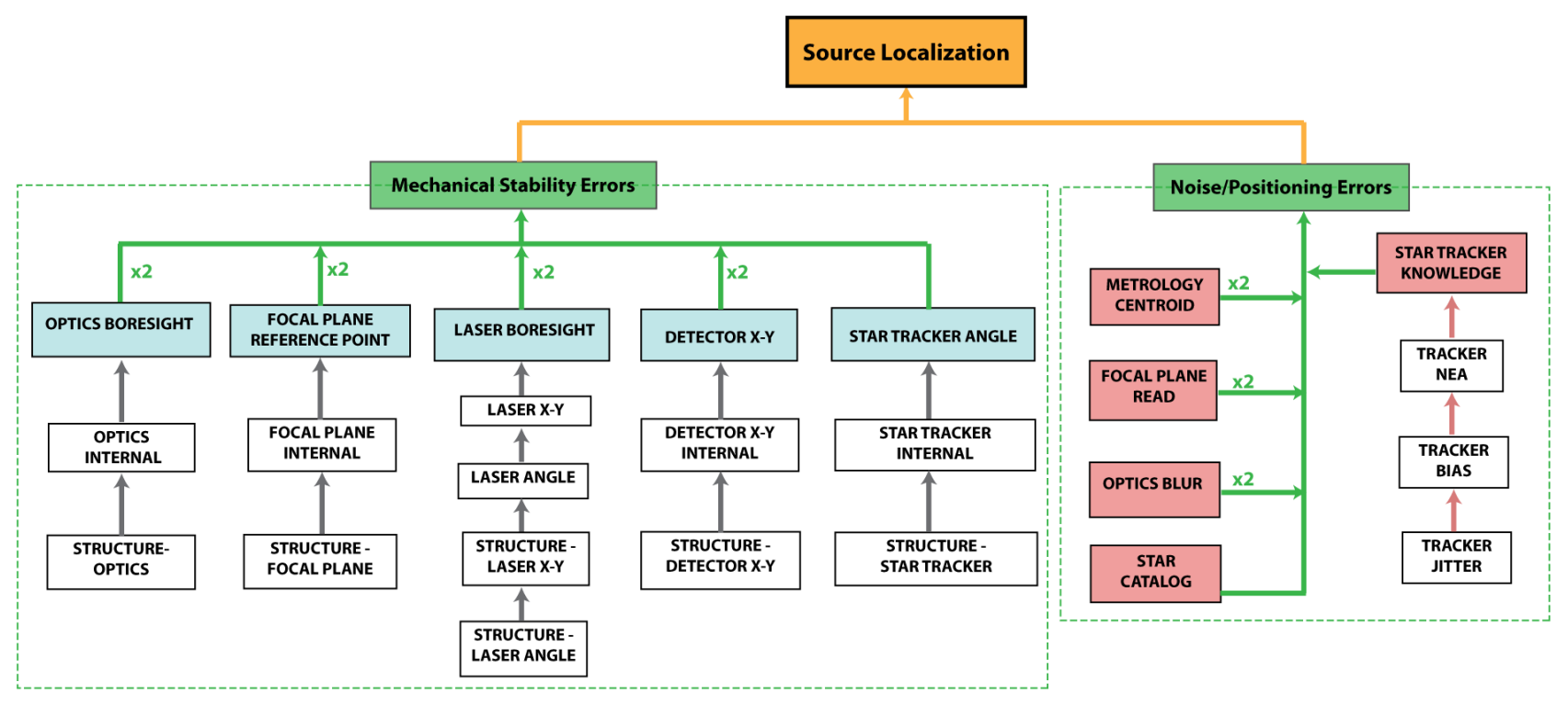

Figure 3. Schematic of the NuSTAR pointing budget for source localization.

${ }^{\Uparrow}$ Developed, refined and utilized in NASA lifecycle phases A, B, and C. Validated in Phase D. 


\subsection{Architecture}

NuSTAR has a science requirement that it shall determine the celestial coordinates of a point source to 10 arcseconds (3 sigma). The pointing budget quantitatively evaluates subsystem performance against this requirement.

To architect the budget, first the instrument design was analyzed for all the error sources in the instrument that could impact source reconstruction. Requirements were written on the instrument to constrain the size of these errors. In continuation, the analysis determined all the subsystem-level errors that contributed to a given instrument error source, and the sizes of those errors were constrained through requirement allocation. The relevant subsystems are: metrology, which includes the star tracker and laser-detector system; structures, which includes the mast; optics; and the focal plane. The pointing budget makes assumptions about how the errors in lower error sets are related to higher sets in order to link the lowest levels to the overall localization requirement. This relationship is illustrated in Figure 3.

\subsection{The Budget}

The NuSTAR pointing budget for source localization in Figure 3 schematizes what was actually a mathematical model implemented in a spreadsheet. The errors are split into two categories: mechanical stability errors, grouped in the green box to the left; and non-mechanical errors, to the right. Instrument (level 3) errors with contributions from multiple subsystems are in colored boxes within the groupings; lower boxes are the contributing subsystem errors. Each arrow in the diagram represents a mathematical operation, whose form will be discussed in 4.3.2.

It should be emphasized that each error box in this diagram corresponds to an actual instrument requirement. After updating the budget with a current best estimate for performance with respect to a given requirement, it yields an immediate and quantitative understanding of how that performance influences overall source localization capability.

\subsubsection{The Terms}

Optics boresight. Only a translation of the center of an optic (its effective pinhole) affects where a photon ends up on the focal plane. The errors associated with the translations of the optics modules are allocated to two subsystems: the center of the optics motion relative to the optics mounting points (optics) and the motion of the optics mounting points relative to the metrology system (structures). The optics are built to minimize those primarily thermal movements, and the optics structure of the observatory is built from composite materials with near-zero CTE to meet stringent thermal distortion requirements.

Focal plane reference point. As long as the angle of the focal plane does not change much, the cosine's foreshortening due to the distortion angle is negligible. This error term therefore refers only to the translation of the center of the focal plane relative to the metrology system. This error term is allocated over the focal plane and structures subsystems: the internal distortions of the focal plane detector, and the distortions in the focal plane. Both of these are driven by thermal changes over the orbit.

Laser boresight. This error term covers how accurately a laser can be pointed at the metrology detectors. Unlike the Xray optics, both angular changes and translations will change the position of the laser spot on the metrology detector. To control the magnitude of (primarily) the angular changes the metrology laser is manufactured from Invar, has an active temperature control system and is covered in 3 thermal shields. Separate requirements on translations and angular changes comprise the overall tolerance, and the instrument allocates requirements to both the structure holding the metrology laser and internal changes in the metrology subsystem.

Detector $x-y$. Similar to the focal plane detector, as long as the angle of the metrology detector does not change much the cosine's foreshortening due to angle is negligible. So only translational error of the metrology detector is allocated over the metrology and the structures subsystems. The metrology detector is built in aluminum, since these stability requirements are considerably less stringent than those of the metrology laser.

Star tracker angle. This is the change in boresight pointing of the instrument star tracker. The absolute position of a star tracker does not matter (because the distance to the stars is so large), but its pointing angle relative to the metrology system does. This error is allocated to angular stability of the optics bench and the internal mechanical pointing stability of the star tracker. 
Metrology centroid. This term refers to how accurately the metrology detector can determine the position of the metrology laser on the metrology detector. This error term includes electronics noise, calibration residual, electronics temperature sensitivity, stray light, contamination, and other smaller error terms.

Focal plane read. There is an error associated with the pixelation of the focal plane detector. This error term also includes calibration errors, since the X-ray pixels are not perfectly gridded. Pixelation errors are significantly less than half a pixel extent, because centroids are calculated over many X-ray events; however, there is also a calibration residual when calculating the centroid.

Optics blur. An X-ray optic does not focus to an infinitesimal spot. The observatory counts single X-ray photons, each of which enters an optic along a slightly different geometric path. Depending on where a photon enters, it will impinge at a different position inside the optic's point spread function. This term characterizes the angular extent of that function.

Star catalog. During the XAO activity previously described, any error in the knowledge of the celestial coordinates of those sources on the sky enters as a bias error into reconstruction.

Star tracker knowledge. This term covers how accurately the star tracker can determine the attitude. It consists of 3 error terms: (1) noise equivalent angle, (2) attitude dependent bias errors (from star tracker catalog errors and internal calibration residual) and (3) errors due to the star tracker's subjection to angular accelerations during mast oscillations.

\subsubsection{Correlation}

The set of errors affecting source localization performance was identified and requirements written to constrain those terms. The terms were then notionally linked to the overall source localization requirement, as illustrated in Figure 3. How this linkage should be specified mathematically raises the necessity of an analysis of correlations.

The most conservative analysis possible assumes that all error terms might be correlated. Addition then links all error terms. However, the conservativeness of this analysis exceeds not only the dictates of engineering pragmatism but also physicality. Most notably, for stability error terms in the NuSTAR instrument system, straight addition suggest motion in opposite directions, because motions in the same direction tend to cancel each other out. For example, if an optic module moves $1 \mathrm{~mm}$, and a focal plane module independently moves $1 \mathrm{~mm}$ in the same direction, the boresight intersection with the focal plane goes unchanged and no corresponding error in source reconstruction appears.

But evidently, in the focal plane there can only ever be two additive terms. If an optic moves left and a focal plane module moves right, then the accumulated error in reconstruction would indeed be the sum of these motions. However, in order to arithmetically add the motion of a metrology laser to these motions, the metrology laser would have to move in an opposite sense of both left and right - clearly impossible.

The laser can, of course, move orthogonally to the line connecting these opposing motions. The mathematical operation of the square root of the sum of squares (RSS) represents this motion. It is popularly said that to RSS errors means they are uncorrelated. While this is true for errors related to distinct physical phenomena (if centroiding noise and laser stability are uncorrelated, the statistical mean of accumulated error will be the RSS of the two errors), for mechanical errors in the NuSTAR system this statement is not entirely accurate.

Because error sources in the same direction always cancel each other out in the reconstruction algorithm, only differences in motion contribute to accumulated error. Thus, on a statistical basis, an RSS of individual errors overpredicts the accumulated error. Physically, for mechanical errors the RSS can be seen as a conservative case under which on average components move orthogonally to one another; for non-mechanical errors, the RSS behaves as it used colloquially: to suggest simple non-correlation.

In brief, for some error sets the RSS is highly conservative and for others it might be somewhat unconservative. For this simple spreadsheet model, it is reasonable to assume that the two balance out. Consequently, a simple RSS of all error terms serves as the nominal case. 


\subsubsection{Results}

As a system engineering exercise in pointing budget analysis, nominal as well as off-nominal correlation scenarios were considered. Estimates for performance were calculated before the builds of flight hardware using the best estimates of requirement performance from design analysis.

The absolute worst case used straight addition of all terms; this assumed positive correlation of all error sources. This analysis yielded an estimate of source localization performance, expressed in terms of reconstructed angular resolution, of about 14 arcseconds against the 10 arcsecond requirement. Because this type of addition implies motion which exceeds the boundaries of 2-dimensional space, it is not particularly meaningful.

An estimate was also computed for a more physical worst case in which addition was allowed wherever it was dimensionally possible, and orthogonality assumed thereafter. This would result, for example, in an optic always moving in the opposite direction as its focal plane; but the line of motion of the metrology laser boresight would have to be orthogonal to that of an optic's boresight. Electronic and noise errors were also assumed to be correlated with thermal stability errors. In this case, the estimate of source localization was about 9 arcseconds, yielding some $10 \%$ margin against the requirement.

However, for reasons discussed above, it was strongly believe that a simple RSS of all terms would be closest to the actual performance of the system. Such an analysis yielded a source localization performance best estimate of 5.5 arcseconds. This estimate maintained $45 \%$ margin against the 10 arcsecond science requirement.

\section{INSTRUMENT SIMULATOR}

\subsection{Concept and Motivation}

While the pointing budget provided system-engineering confidence that the design of the instrument would yield adequate science performance, the prevalence of assumptions and simplifications in that early model made it insufficiently rigorous for formal verification of science requirements. An analysis tool was needed that either validated the assumptions of the pointing budget, or verified science requirements directly.

Given the budgetary constraints of a small project, construction of a new, elaborate model of the observatory's behavior was not practical. Instead, a number of independent models and analyses used to predict performance for subsystems would be combined in a common code and interfaced realistically. By modeling all of the dominant thermo-structural, optical, and metrological activities and interactions of the observatory, an end-to-end mission would be simulated. This simulation would fulfill the following objectives:

1. Validation of the pointing budget and its driving assumptions.

2. Direct verification of level two science requirements on reconstructed angular resolution.

3. Demonstration that the photon source reconstruction and XAO algorithms (see section 3) are correct, and that their error contributes negligibly to angular resolution.

4. Generation of realistic sample data for the data analysis pipeline.

\subsection{The Code}

This NuSTAR simulator, dubbed NuSim, was written in C++ utilizing CERN's ROOT software library. ${ }^{6}$ It uses ROOT's graphical user interface and data display functionality, along with some high level support classes (strings, rotations, etc.).

NuSim was implemented in a completely object-oriented way with the aim to separate data from the simulation tasks. Moreover, each simulation task (such as the passing of photons through an optic, or the interactions of these X-rays with a detector) was written as an independent module; interfaces were then standardized. Special functions common to many 
modules (such as photon propagation) were implemented in decorator classes from which the affected module classes were derived.

As a consequence of this highly modular architecture, different scientists and engineers can easily write and maintain the various modules; only a few functions - the initialization, the actual analysis function, the finalization, and the loading and saving user data - require the individual module designer. A supervisor takes care of the order of simulations, the initialization and finalization of the modules, the propagation of data from module to module, the progress of time, the stop conditions, and any user interactions.

NuSim has several features that allow the photon data and sensor data to be saved at different locations. It also has the option of saving data into actual flight format for testing with the ground data system pipeline. The architecture is very flexible and with the data files read in as configuration-controlled database it can easily and reliably be updated.

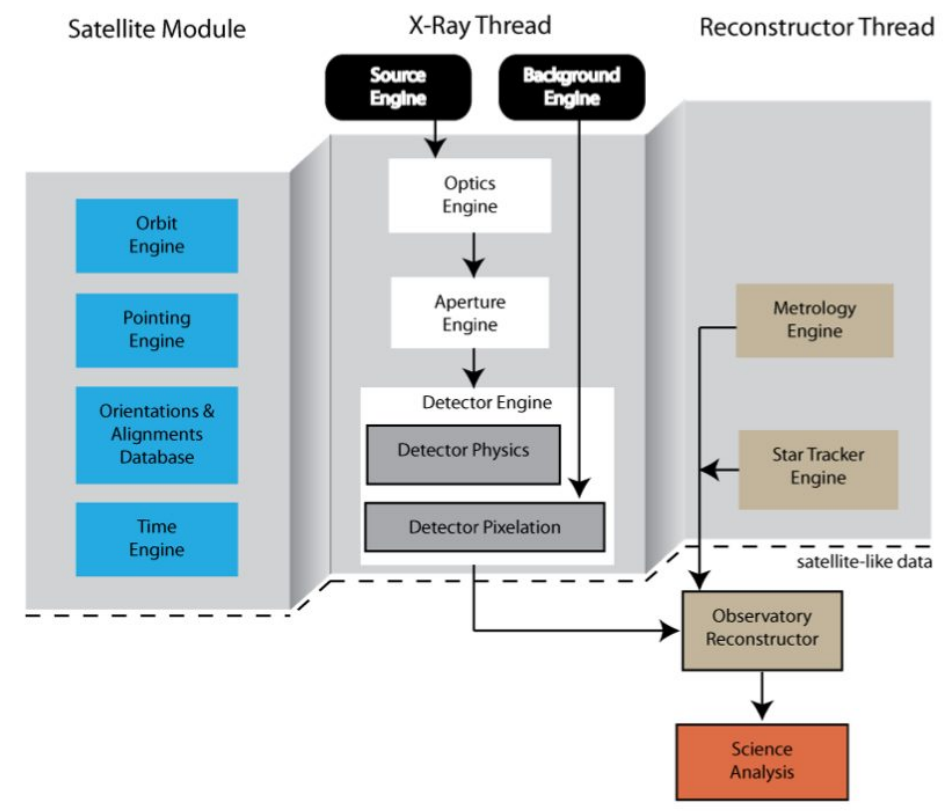

Figure 4. Schematic of the NuSim architecture.

\subsection{Design and Architecture}

The NuSim architecture was driven by the need for a realistic geometric representation of the instrument and photon path, along with a lightweight and modular code, to allow the comprehensive studies of the effects of perturbations necessary to validate the overall pointing budget.

A second drive on the design was the need to recreate the onboard data flow of sensor and photon data and to simulate their interaction, since ultimately the metrology laser data determines the spatial extent of the reconstructed photon data.

Figure 4 schematizes the NuSim architecture. The leftmost panel of the diagram, the "Satellite Module" represents the NuSim supervisor control and all the input databases. The most critical input to this module, in the context of accurately recreating satellite source localization performance is the orientations and alignments database, which contains timestamped 6-DOF transformations between all the modeled instrument components. The transformations are stored both in ideal configuration and perturbed over an orbit, as derived from detailed thermal analyses of the observatory. The pointing and orbit engines describe the spacecraft orbit location and inertial pointing, as derived from GNC analyses of the spacecraft that correspond to the same thermal cases used to produce the orientations databases.

The "X-Ray Thread" represents the photon path. A flexible source engine can produce a variety of source spectrums and morphologies. The created photons are sent into the optics engine, which is a Monte Carlo ray-trace. The ray-trace reads 
external reflectivity files which can easily be updated, and includes obscuration and scattering in the optics. The photon is passed down the path, subjected to any attenuations and obscurations, and then enters the detector engine. The detector engine realistically simulates the physics of the photon interaction in CdZnTe detectors. Lookup tables unique to the individual detectors solve for the redistribution and splitting of charge by an interaction due to a pixel grid.

The rightmost panel represents the sensor data path. The metrology sensor data is produced in NuSim, at the same rate as in orbit, by simply noting the instantaneous alignment of the system and calculating the laser intersection at the metrology detector. NuSim also generates the star tracker camera quaternion at the same rate as it will be in orbit, using the instantaneous pointing direction of the outboard bench from the satellite module. There are Monte Carlo errors appropriate to subsystem performance estimates associated with each sensor datum.

The observatory reconstructor module combines the photon data stream with the sensor data stream, using the algorithms presented in section 3. It is shown in Figure 4 independently at the bottom. The module has been written in such a way that it can be used as standalone program; at a later date, when the algorithms have been validated, it can be used without modification in the event reconstruction pipeline during flight.

\section{SUMMARY}

This paper has discussed how the celestial coordinates of an X-ray source are determined by the NuSTAR observatory, seen from a systems-engineering perspective. Initially, an error budget was constructed. The error budget was used to identify the need for a metrology system to achieve the required reconstructed source localization knowledge. The same budget was then used to allocate errors to the different subsystems. For requirements validation, a software simulator is being used to prove that individual subsystem performance adds up to the levied science requirements. Equations for transforming X-ray photon impingements on the X-ray detector into celestial coordinates using the metrology system, and the on-orbit calibration activity associated with that transformation, have also been discussed.

\section{ACKNOWLEDGEMENTS}

The research described in this paper was carried out at the Jet Propulsion Laboratory, California Institute of Technology, Space Radiation Laboratory, California Institute of Technology and Space Sciences Laboratory, UC Berkeley and was sponsored by the National Aeronautics and Space Administration. References herein to any specific commercial product, process or service by trademark, manufacturer, or otherwise, does not constitute or imply its endorsement by the United States Government or the Jet Propulsion Laboratory, California Institute of Technology, the Space Radiation Laboratory, California Institute of Technology, or the Space Sciences Laboratory, UC Berkeley

\section{REFERENCES}

[1] Orbital Sciences Corporation, "Fact Sheet: LEOStar ${ }^{\mathrm{TM}}$." $<$ http://www.orbital.com/NewsInfo/Publications/LEOStar_Fact.pdf> Accessed 11 May 2010.

[2] Mao, P.H., Harrison, F.A., Windt, D.L., and Christensen, F.E., "Optimization of graded multilayer designs for astronomical x-ray telescopes,” Applied Optics 38(22), 4766-4775 (1999).

[3] Liebe, C.C., "Accuracy performance of star trackers: a tutorial," IEEE Transactions on Aerospace and Electronic Systems 38(2) 587-599 (2002).

[4] Liebe, C.C., et al, "Metrology system for measuring mast motions on the NuSTAR mission," Accepted to Proc. IEEE, March 2010.

[5] Horn, B.K.P., "Closed-form solution of absolute orientation using unit quaternions," Journal of the Optical Society of America A 4, 629-642 (1987).

[6] Brun, R., and Rademakers, F., "ROOT - An Object Oriented Data Analysis Framework", NIM A 389 (1997) 81-86. See also http://root.cern.ch/. 